\title{
A study of timing properties of Silicon Photomultipliers
}

\author{
Paola Avella ${ }^{\mathrm{a}, *}$, Antonella De Santo ${ }^{\mathrm{b}}$, Annika Lohstroh $^{\mathrm{a}}$, Muhammad T. Sajjad ${ }^{\mathrm{a}, \mathrm{c}}$, Paul J. Sellin ${ }^{\mathrm{a}}$ \\ ${ }^{a}$ Department of Physics, University of Surrey, Guildford, GU2 7XH, Surrey, UK, South East Physics Network (SEPNet) member \\ ${ }^{b}$ Department of Physics and Astronomy, University of Sussex, Brighton, BN1 9QH, Sussex, UK, South East Physics Network (SEPNet) member \\ ${ }^{c}$ Advanced Technology Institute, University of Surrey, Guildford, GU2 7XH, Surrey, UK
}

\begin{abstract}
Silicon Photomultipliers (SiPM) are solid-state pixelated photodetectors. Lately these sensors have been investigated for Time of Flight Positron Emission Tomography (ToF-PET) applications, where very good coincidence time resolution of the order of hundreds of picoseconds imply spatial resolution of the order of $\mathrm{cm}$ in the image reconstruction. The very fast rise time typical of the avalanche discharge improves the time resolution, but can be limited by the readout electronics and the technology used to construct the device. In this work the parameters of the equivalent circuit of the device that directly affect the pulse shape, namely the quenching resistance and capacitance and the diode and parasitic capacitances, were calculated. The mean rise time obtained with different preamplifiers was also measured.
\end{abstract}

Keywords: ToF-PET, SiPM, MPPC, pulse shape, SiPM parameters

\section{Introduction}

ToF-PET is a medical diagnostic technique that gives infor- ${ }^{27}$ mation on the metabolic activity of cells. It requires the use of ${ }^{28}$ detectors with a very fast response, that in turn determines very ${ }^{29}$ good time resolution. Being analogue to the Photomultiplier ${ }^{30}$ Tubes (PMTs), traditionally used for PET applications, the Sil- ${ }^{31}$ icon Photomultiplier (SiPM or MPPC) is currently one of the ${ }^{32}$ most promising sensors for ToF-PET, in association with very ${ }^{33}$ fast scintillating crystals. The main improvements in the use of ${ }^{34}$ SiPMs are expected to be compactness of the designs and a su- ${ }^{35}$ perior coincidence time resolution. Moreover, its insensitivity ${ }^{36}$ to magnetic fields opens the horizons toward the combination ${ }^{37}$ of the ToF-PET with the Nuclear Magnetic Resonance (NMR). ${ }^{38}$

Silicon Photomultipliers are solid-state single-photon sensi- ${ }^{39}$ tive devices made of a matrix of Geiger Mode Avalanche Photo- ${ }^{40}$ diodes (GM-APD). Each pixel works as a binary device, giving ${ }^{41}$ in output a standard signal. The almost simultaneous firing of ${ }^{42}$ multiple pixels preserves the information on the number of pho- ${ }^{43}$ tons striking the sensor. However, due to the limited number of ${ }^{44}$ pixels in the device, saturation effects can affect the linearity of ${ }^{45}$ its response, whenever the one-to-one correspondence between ${ }^{46}$ a photon and a pixel is lost; i.e. the multiple photons incident ${ }^{47}$ on the same pixel at the same time generate the same standard output as a single detected photon [1].

\footnotetext{
${ }^{*}$ Corresponding author

Department of Physics

Faculty of Engineering and Physical Sciences

University of Surrey

Guildford, GU2 7XH

Surrey, UK

Tel: +44 (0) 1483682697

Email address: p.avella@surrey.ac.uk (Paola Avella)
}

The formation of the signal is generated by the photoelectric effect of visible photons in the Silicon bulk. The detector is reverse biased at a voltage higher than the breakdown voltage, $\mathrm{V}_{b r}$ and the carriers migrate to a region with a very high electric field of the order of $10^{5} \mathrm{~V} \mathrm{~cm}^{-1}$. Once here these carriers have enough energy to create an electron-hole pair by impact ionisation. The daughter carriers in turn are accelerated by the strong electric field and will generate further carriers. An avalanche effect is settled in the very thin depletion region of the sensor of the order of few $\mu \mathrm{m}$, characterised by a current of the order of $\mu \mathrm{A}$ [2]. This avalanche is passively quenched by the high resistivity of a resistor $\mathrm{R}_{q}$, in series with each pixel. The presence of $\mathrm{R}_{q}$ contributes to the pulse formation with a very small capacitance in parallel, $\mathrm{C}_{q}$. The typical pulse shape of a SiPM is then characterised by the build-up time of the avalanche, of the order of tens of $\mathrm{ps}$, and by a mixed contribution from $\mathrm{R}_{q}, \mathrm{C}_{q}$ and the diode capacitance $\mathrm{C}_{d}$, that affect mainly the decay time of the pulse, of the order of tens of ns. Finally, the readout electronics can play a degrading role on the timing performances of these devices, in particular affecting the pulse rise time.

In this paper, the characterisation of various MPPCs will be discussed and the effects of different readout electronics will be studied.

\section{Experimental method}

The experiments described in this work were performed using four different Hamamatsu MPPC, S10362-11-025C, S10362-11-50C, S10362-33-025C and S10362-33-050C, with a total area of $1 \times 1 \mathrm{~mm}^{2}$ and $3 \times 3 \mathrm{~mm}^{2}$ and pixel size of $25 \mu \mathrm{m}$ and $50 \mu \mathrm{m}$ (cfr. Tab.1).

The IV forward and reverse characteristics were measured using a Keithley 487 picoammeter interfaced with a computer 


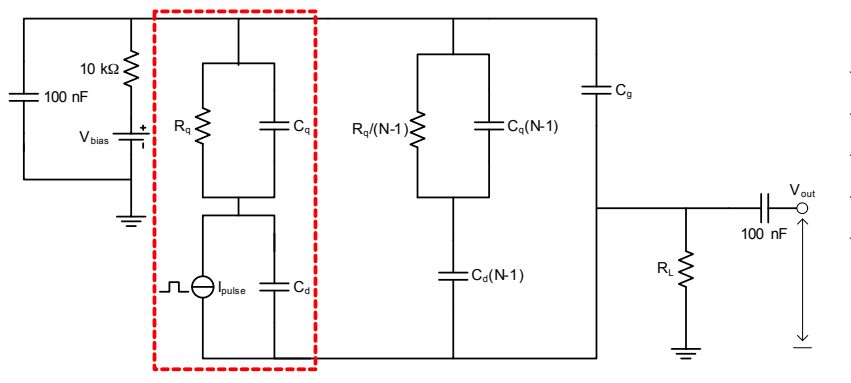

Figure 1: SiPM equivalent circuit with bias circuit suggested by Hamamatsu [6]. The portion in the red dashed square indicates one pixel firing.

through a dedicated LabVIEW DAQ software. The capacitance ${ }_{105}$ and conductance of the sensors were instead measured using an ${ }_{106}$ Agilent 4284A LCR meter at $1 \mathrm{~V}$ AC voltage and at a frequency ${ }_{107}$ of $1 \mathrm{MHz}$ as in [3][4][5].

The values of $\mathrm{R}_{q}$ and of $\mathrm{V}_{b r}$ were calculated from the IV- ${ }_{-109}^{108}$ characteristics. A linear fit to the Ohmic part of the forward ${ }_{110}$ curve gives the total SiPM resistance, from which it is pos- ${ }_{111}$ sible to calculate $\mathrm{R}_{q}$ considering that the pixels are in paral- ${ }_{-12}$ lel. A parabolic fit to the region between the proportional and ${ }_{113}$ the avalanche part of the reverse curve, gives instead the value ${ }_{114}$ of $\mathrm{V}_{b r}$, which is needed to correctly operate the device in the ${ }_{115}$ Geiger mode. The values found are summarized in Tab.1.

For the measurement of the charge released by dark pulses, 117 an Hamamatsu Si $p-i-n$ diode S1223 was used for normal-118 isation purposes, readout by a current amplifier Ortec VT 120.119 The pulses were acquired and stored by means of a dedicated ${ }_{120}$ LabVIEW DAQ software by a Tektronix TDS 7254B digital ${ }_{12}$ Phosphor oscilloscope, with a sampling rate of $20 \mathrm{GS} / \mathrm{s}$ and 2.5 GHz bandwidth. An Ortec 710 quad 1-kV bias supply was used to bias the $p-i-n$ diode and the MPPCs respectively.

The equivalent circuit of a generic SiPM has been devel-123 oped recently by Corsi [3]. Fig.1 shows this circuit along with ${ }_{124}$ the bias filter, the load resistor $\mathrm{R}_{L}$ and the coupling capacitor. ${ }_{125}$ In this figure the dashed rectangle represents a single-photon event: the current due to the avalanche is reproduced by a current pulse with a maximum value of $20 \mu \mathrm{A}$ and arbitrary short duration of few ps.

For the calculation of the charge developed by a single avalanche event, the pulse generated by the interaction of an $\alpha$ particle from the decay of ${ }^{241} \mathrm{Am}$ in the Si bulk of a $p-i-n$ diode was used for normalization purposes. Considering a distance between the $\alpha$-particle and the entrance window of the diode of about $3 \mathrm{~mm}$, and the attenuation factor of $\alpha$-particles in air equal to $8.59 \times 10^{-2} \mathrm{MeV} / \mathrm{mm}$ [7], an uncertainty of $0.1 \mathrm{MeV}$ was attributed to the energy of the source, $\mathrm{E}_{\alpha}=5.3 \pm 0.1 \mathrm{MeV}$. Taking into account the energy needed to create an electronhole pair in $\mathrm{Si}, \epsilon_{i}=3.62 \mathrm{eV}$, the contribution of e-h pairs to the total charge is $Q_{p i n}=240 \pm 30 \mathrm{fC}$. The charge released by a dark pulse was then obtained from the relation $Q_{S i P M}=Q_{p i n} \cdot x_{c}^{S i P M} / x_{c}^{p i n}$, where $x_{c}^{p i n}$ and $x_{c}^{S i P M}$ represent the centroid of the distribution of the integral over the same number of pulses for the $p-i-n$ diode and the SiPM respectively. The slope of the curve of single-photon charge versus the bias voltage gives the value of the pixel capacitance $\mathrm{C}_{\text {pixel }}$ (see Tab.1). From the calculation of the total admittance of the circuit, and using the values of the capacitance $\mathrm{C}_{\text {meas }}$ and conductance $\mathrm{G}_{\text {meas }}$ measured with the LCR meter, the values of the diode, quenching and parasitic capacitance of the circuit in Fig.1 are found solving the system [8]:

$$
\begin{aligned}
& C_{d}=\sqrt{G_{\text {meas }} \cdot \frac{1+\omega^{2} R_{q}^{2} C_{\text {pixel }}^{2}}{N_{\text {pixel }} \omega^{2} R_{q}^{2}}} \\
& C_{q}=C_{\text {pixel }}-C_{d} \\
& C_{g}=C_{\text {meas }}-N_{\text {pixel }} C_{d}+\frac{\omega^{2} N_{\text {pixel }} R_{q}^{2} C_{d}^{2} C_{\text {pixel }}}{1+\omega^{2} R_{q}^{2} C_{\text {pixel }}^{2}} .
\end{aligned}
$$

For the study of the effects of different readout electronics on the pulse shape, the MPPCs were illuminated by a Ti:Sapphire Coherent ${ }^{\mathrm{TM}}$ ultra fast laser system giving in output about 100 fs pulses centered at $800 \mathrm{~nm}$ with a repetition rate of $250 \mathrm{kHz}$. The experimental setup is shown in Fig.2. A mirror chain was used to direct the light beam on the sensor and a beam splitter was used to further reduce the intensity of the light. Thorlabs reflective neutral density filters with various optical densities were used to modulate the light intensity. An aperture was used to reduce the number of photons hitting the devices and the detector was housed in an Al box with a collimator entrance window to further reduce background light contamination. The multiple-photon signal (typically containing less than 50 photons) was readout by three commercial current preamplifiers, Ortec VT 120, GSI DBA IV/R and Mini-Circuits ZFL-1000 LN, with same $50 \Omega$ input impedance, but different bandwidth, gain and noise performances (cfr. Tab.2).

\section{Results and Discussion}

\subsection{The MPPC characterisation}

The method described in Section 2 allows the calculation of the values of the electric parameters of the device, namely

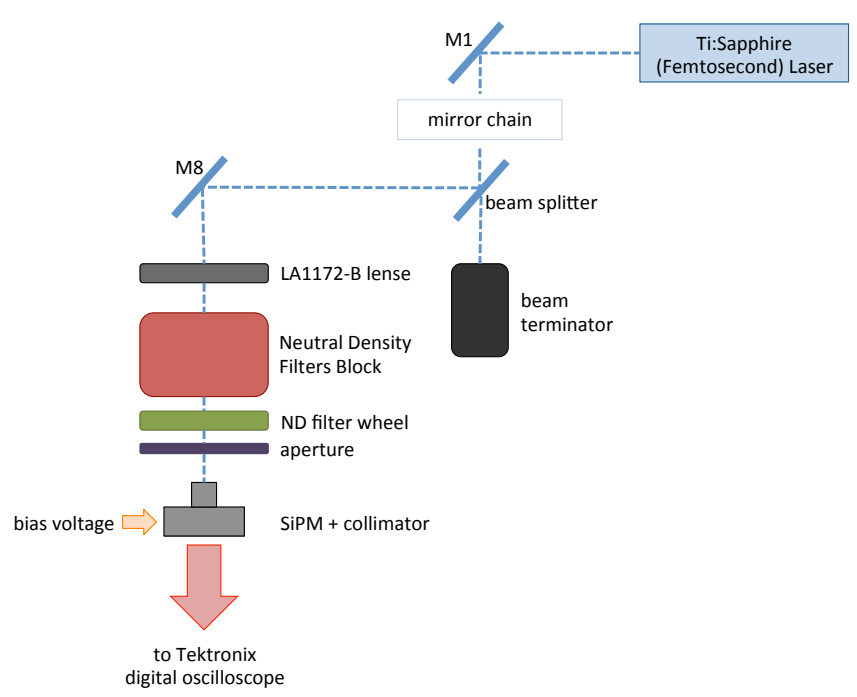

Figure 2: Setup for the test of the readout electronics. 


\begin{tabular}{lcccc}
\hline Parameters & $\mathbf{1 1 - 2 5 C}$ & $\mathbf{3 3 - 2 5 C}$ & $\mathbf{1 1 - 5 0 C}$ & $\mathbf{3 3 - 5 0 C}$ \\
\hline Total Area $\left(\mathrm{mm}^{2}\right)$ & $1 \times 1$ & $3 \times 3$ & $1 \times 1$ & $3 \times 3$ \\
Pixel Size $(\mu \mathrm{m})$ & 25 & 25 & 50 & 50 \\
$\mathrm{~N}_{\text {pixel }}$ & 1600 & 14400 & 400 & 3600 \\
$\mathrm{~V}_{\text {br }}(\mathrm{V})$ & $68.31 \pm 0.06$ & $69.25 \pm 0.08$ & $68.138 \pm 0.006$ & $69.567 \pm 0.004$ \\
$\mathrm{R}_{q}(\mathrm{k} \Omega)$ & $218.0 \pm 0.5$ & $358.1 \pm 0.7$ & $131 \pm 4$ & $163.1 \pm 0.4$ \\
$\mathrm{G}_{\text {meas }}(\mu \mathrm{S})$ & $3.064 \pm 0.008$ & $46.84 \pm 0.08$ & $10.40 \pm 0.08$ & $139.9 \pm 0.4$ \\
$\mathrm{C}_{\text {meas }}(\mathrm{pF})$ & $32.82 \pm 0.04$ & $272.0 \pm 0.3$ & $36.72 \pm 0.06$ & $304.1 \pm 0.6$ \\
$\mathrm{C}_{q}(\mathrm{fF})$ & $2.9 \pm 0.9$ & $2.5 \pm 0.6$ & $7 \pm 6$ & $7 \pm 8$ \\
$\mathrm{C}_{d}(\mathrm{fF})$ & $14.92 \pm 0.02$ & $15.20 \pm 0.02$ & $71 \pm 1$ & $78.0 \pm 0.2$ \\
$\mathrm{C}_{g}(\mathrm{pF})$ & $8.97 \pm 0.06$ & $53.4 \pm 0.4$ & $8 \pm 2$ & $25.3 \pm 0.9$ \\
$\mathrm{C}_{\text {pixel }}(\mathrm{fF})$ & $17.8 \pm 0.9$ & $16.7 \pm 0.6$ & $78 \pm 6$ & $85 \pm 8$ \\
\hline
\end{tabular}

Table 1: Table of the measured Hamamatsu MPPC parameters.

\begin{tabular}{lcccc}
\hline Preamplifier & $\begin{array}{c}\text { Bandwidth } \\
(\mathrm{MHz})\end{array}$ & $\begin{array}{c}\text { Gain } \\
(\mathrm{dB})\end{array}$ & $\begin{array}{c}\text { Rise time } \\
\text { MPPC 11-25C (ns) }\end{array}$ & $\begin{array}{c}\text { Rise time } \\
\text { MPPC 33-25C (ns) }\end{array}$ \\
\hline Ortec VT 120 & $10-350$ & 46 & $1.714 \pm 0.001$ & $3.513 \pm 0.001$ \\
Mini-Circuits ZFL & $0.1-1000$ & 20 & $1.916 \pm 0.002$ & $3.732 \pm 0.001$ \\
GSI DBA IV/R & $0.003-2000$ & $23-45$ & $1.988 \pm 0.001$ & $3.399 \pm 0.002$ \\
No preamp & - & - & $2.223 \pm 0.002$ & $4.148 \pm 0.004$ \\
\hline
\end{tabular}

Table 2: Table of the used current amplifiers and the measured rise times.

$\mathrm{C}_{\text {pixel }}, \mathrm{C}_{d}$ and $\mathrm{C}_{q}$. These values are summarized in Tab.1. It is found that for devices with the same pixel size, the values of $\mathrm{C}_{d}$ are of the same order of magnitude, consistent with the values reported in [4] and [5]. However, the small discrepancy found for devices with same pixel size, but different total area, can be explained by a strong dependency of the gain and $R_{q}$, primarily, on the temperature. Small variations in room temperature are not included in the quoted uncertainties in Tab.1. In the case of $\mathrm{C}_{q}$, the values are consistent for devices with the same pixel size. Finally, it results that for devices with same total area, the value of $\mathrm{C}_{g}$ is smaller in the case of the MPPCs with a bigger pixel size. In fact, the parasitic capacitance is associated to the grid that connects the pixels in parallel, and this grid is more dense in the case of $25 \mu \mathrm{m}$ pixel MPPCs. The results obtained are in agreement with this.

\subsection{The effects of the readout electronics}

The knowledge of the sensor parameters allows a SPICE (Simulation Program with Integrated Circuit Emphasis) simulation of the device. Fig. 3 shows the voltage pulses obtained ${ }^{15}$ from the simulation of the MPPC under study, taking into ac- ${ }^{159}$ count only $R_{L}$ and the coupling capacitance and ignoring any 160 additional external amplification circuit. The $10 \%$ to $90 \%$ total ${ }^{161}$ amplitude rise times are below $30 \mathrm{ps}$ for all the devices. How-162 ever, the simulated results for the MPPCs $3325 \mathrm{C}$ and $3350 \mathrm{C}^{163}$ give an overall slower signal. This is due to a combined effect ${ }_{164}$ of $C_{q}, C_{g}$ and $R_{L}$ on the rise time and $C_{d}$ and $R_{q}$ on the decay 165 time [3]. These results are far from the rise time values ob-166 tained with three different current preamplifiers measured using ${ }_{167}$ the setup in Fig.2. Fig.4 shows the histogram of the rise time for 168 the MPPC 11-25C. This discrepancy between the simulated and ${ }_{169}$ the measured rise time could well be due to the fact that in the 170 simulated case a single photon pulse, while in the experimental ${ }_{171}$

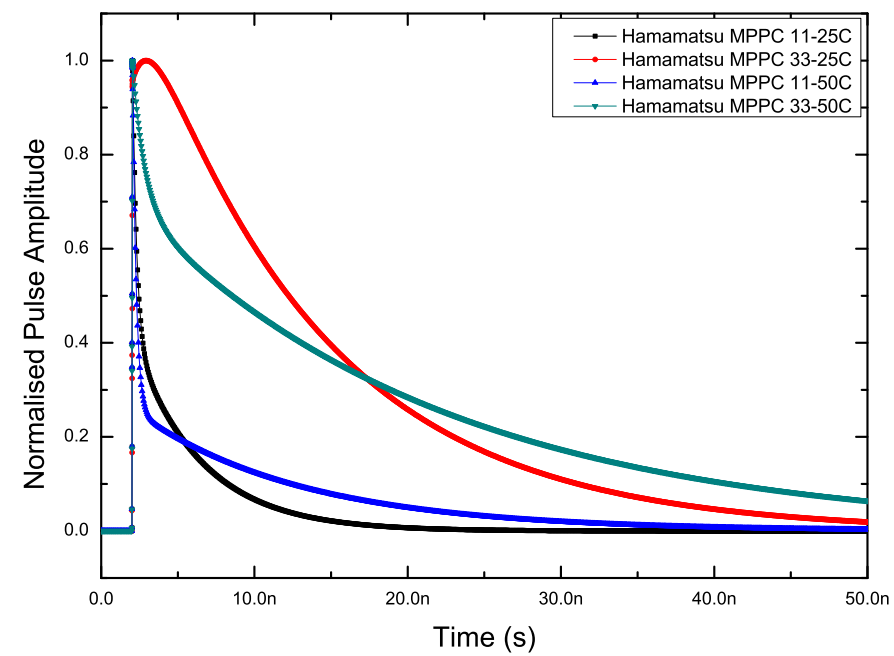

Figure 3: SiPM pulses from a SPICE simulation using the parameters calculated in Tab.1.

results multiple-photon pulses were considered. The results are summarised in Tab. 2 for both $25 \mu \mathrm{m}$ sensors.

The rise time, signal to noise ratio (SNR) and time jitter of multiple-photon pulses were studied using the femto-laser setup. Fig.5 shows that the rise time deteriorates when the bias voltage is increased in the case of the 11-25C MPPC.

This trend corresponds to an increase of dark noise, i.e. to an increase in the number of afterpulses and hot carriers with bias voltage, over the signal level. Fig.5 shows that the time jitter, defined as $\sigma_{j}=t_{r} / S N R$, where $\mathrm{SNR}=V_{\text {pulse }}^{r m s} / \sigma_{\text {noise }}^{r m s}$, improves with overvoltage. In fact, the increase in the bias voltage improves the SNR of the sensor at fixed light intensity. The high frequency noise that affects the signal and is significant at lower bias voltage, becomes less significant due to the higher influ- 


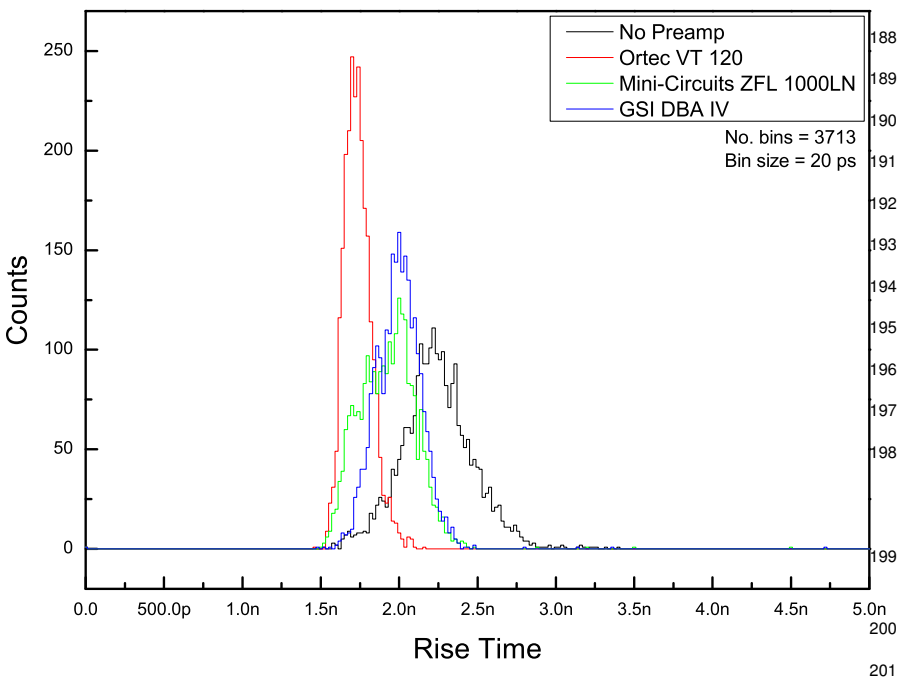

Figure 4: Histogram of the rise time obtained using the Hamamatsu MPPC $11-25 \mathrm{C}$ and the femto-laser.

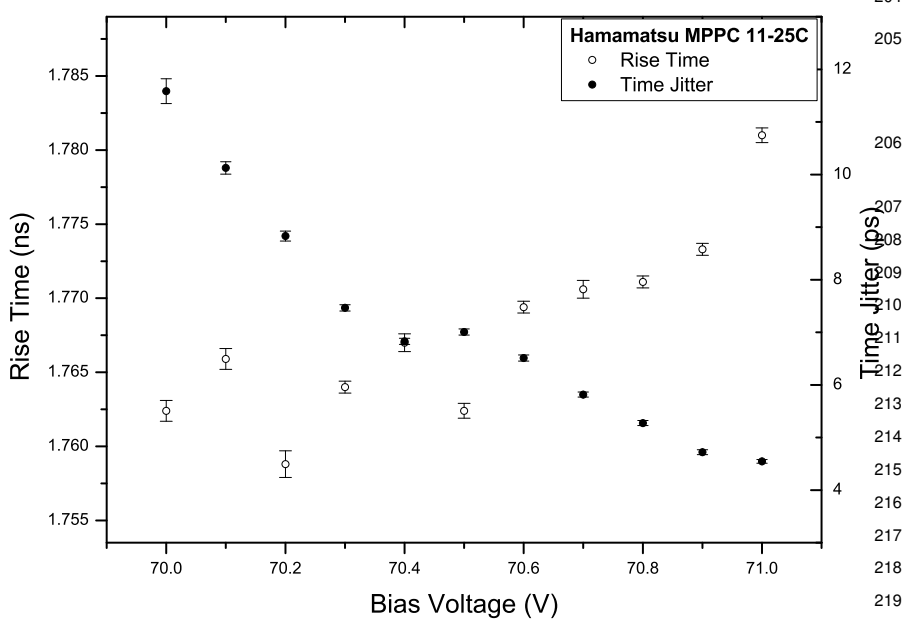

design of a custom-made bias circuit. From the measurement of the rise time it is also found that the time jitter improves with increasing bias voltage due to the corresponding increase in the SiPM gain and a resulting improvement of the SNR. Then these devices do not need to be readout with very fast amplifiers. However, current amplifiers with good noise performances are required to achieve good performances in timing applications. Then, with the knowledge of the terminal capacitance of these devices it is possible to construct custom made preamplifiers with the right value of matching impedance and a good compromise between amplification and SNR.

\section{Acknowledgements}

The authors are very thankful to the South East Physics Network (SEPNet) for financial support of the work described in this document. They are also grateful to B. Camanzi from Rutherford Appleton Laboratory and to I. Marko from Advanced Technology Institute, University of Surrey, for access to electronics used in the experiments and for useful discussions.

\section{References}

[1] J. Haba, Nucl. Instr. Meth., A595 (2008) 154-160;

[2] W. T. Tsang, Semiconductors and Semimetals, vol. 22, Lightwave Communications Technology, Part D, Photodetectors, Academic Press Inc, 1985 ;

[3] F. Corsi et al., Nuclear Science Symposium Conference Record, 2006. IEEE, vol. 2, 2006;

[4] K. Yamamoto, NIMA (2010), doi:10.1016/j.nima.2010.04.112;

[5] S. Seifert et al., Nuclear Science, IEEE Transactions on , vol. 56, no. 6, pp. 3726-3733, Dec. 2009;

[6] Hamamatsu Photonics K. K., MPPC Technical Information, Application Notes;

[7] S. Gkoumas, PhD Thesis, University of Surrey, UK, 2011;

[8] F. Corsi et al., NIMA 572 (2007) 416-418.

Figure 5: Measured rise time and time jitter for the Hamamatsu MPPC 11-25C at fixed light intensity and readout without any preamplifier.

ence of the pulse amplitude on the SNR and subsequently on the time jitter.

\section{Conculsions}

In this paper the parameters of different MPPCs were calculated. A good agreement with the results obtained in previous characterisation [4][5] was obtained. A SPICE simulation of the equivalent circuit showed a very fast pulse, meaning that the effective rise time of the output signal of these sensors is deteriorated mainly by the readout electronics. For this reason, measurements of the rise time using several commercial current preamplifiers with different bandwidth, gain and noise figure were performed. It resulted that the use of a poor quality circuitry (i.e. coaxial components and standard plain PCB) deteriorates the rise time. This is due to parasitic inductances and impedances mismatch. Future developments will be the upgrade of the circuitry to high quality components as well as the 